\title{
Magnetic V embedded in copper evidenced by x-ray magnetic circular dichroism
}

\author{
Y. Huttel, ${ }^{1, *}$ G. van der Laan,,${ }^{2, \dagger}$ C. M. Teodorescu, ${ }^{2}$ P. Bencok, ${ }^{3}$ and S. S. Dhesi ${ }^{3}$ \\ ${ }^{1}$ Instituto de Microelectrónica de Madrid, CNM, CSIC, 28760 Tres Cantos, Madrid, Spain \\ ${ }^{2}$ Magnetic Spectroscopy Group, Daresbury Laboratory, Warrington WA4 4AD, United Kingdom \\ ${ }^{3}$ European Synchrotron Radiation Facility, Grenoble, France \\ (Received 13 December 2002; published 27 February 2003)
}

\begin{abstract}
We report the observation of a magnetic moment on vanadium embedded in copper. Using $\mathrm{x}$-ray-absorption spectroscopy and $\mathrm{x}$-ray magnetic circular dichroism we show that the vanadium atomic magnetic moment is strongly dependent on the formation temperature of the $\mathrm{VCu}$ alloy. The absorption spectra allow us to distinguish the electronic transitions which are the signature of the magnetic state from the nonmagnetic states. We discuss the temperature dependence of the magnetic moment of vanadium and show that alloys prepared at 250 and $270 \mathrm{~K}$ do follow a ferrimagnetic behavior rather than a Curie-Weiss law.
\end{abstract}

DOI: 10.1103/PhysRevB.67.052408

PACS number(s): 75.75.+a, 75.50.Ss, 75.70.Ak, 78.70.Dm

The study of the magnetic properties of vanadium in other forms than its well known bcc paramagnetic structure such as in free and supported clusters, surfaces, multilayered compounds and alloys is subject of intense debate and extensive research. For example, vanadium on surfaces has been predicted to be nonmagnetic, ${ }^{1}$ ferromagnetic, ${ }^{2}$ antiferromagnetic, ${ }^{3-5}$ and paramagnetic, ${ }^{6}$ whereas experimental results evidence ferromagnetic, ${ }^{7}$ antiferromagnetic, ${ }^{8,9}$ and nonmagnetic ${ }^{10,11}$ behavior. Also the results for the magnetic properties of small $\mathrm{V}$ clusters ${ }^{1,8,12-14}$ are contradictory while very little is known about the magnetic properties of $\mathrm{V}$ compounds and alloys. ${ }^{15}$ It has been shown that the magnetic properties of vanadium on surfaces, in clusters and alloys are strongly related to the local geometry (i.e., coordination number and neighbor distances) $)^{1,12-14,16}$ and the resulting electronic structure. This dependency is such that the vanadium, being nonmagnetic in its bulk form, could become magnetic when associated with other nonmagnetic elements such as copper. The theoretical prediction of the violation of Hund's third rule and antiparallel V spin and orbital moments in $\mathrm{VAu}_{4}$ alloy ${ }^{17}$ together with the discrepancy between different theoretical approaches ${ }^{18,19}$ have made the magnetic properties of $\mathrm{V}$ compounds and alloys one of the challenging problems in solid-state physics and material science. Most surprising are the strongly enhanced magnetic moments of vanadium impurities in thin films of sodium and potassium as measured by anomalous Hall effect. ${ }^{20}$ The understanding and tailoring of the magnetic properties of small particles for their further integration into magneto-optical and highdensity data storage devices ${ }^{21}$ is an important motivation for the study of $\mathrm{VCu}$ compounds since their magnetic properties might fit the sought after requirements for incorporation into modern magnetic devices. As a consequence, there is an urgent need of new studies and in particular new experimental results on the magnetic properties of $\mathrm{V}$ systems. ${ }^{17-19}$

In this report we present an X-ray-absorption spectroscopy (XAS) and X-ray magnetic circular dichroism (XMCD) study of $\mathrm{VCu}$ alloy. We show that depending on the deposition temperature, different $\mathrm{V}$ species can be formed and that the magnetic properties of the alloy strongly depend on the presence of some of these species. We find that the temperature dependence of the magnetic moment does not follow a (super)paramagnetic law but rather a ferrimagnetic behavior.

$\mathrm{VCu}$ alloys were prepared in an ultrahigh vacuum (UHV) preparation chamber operating in the $10^{-10}$ mbar. Controlled coevaporation of vanadium and copper was performed on a $\mathrm{Cu}(100)$ surface cleaned by standard cycles of $\mathrm{Ar}^{+}$sputtering and annealing. The stoichiometry was controlled in situ by measuring the evaporation rate of both the copper and the vanadium evaporator. The calibration was performed using a quartz microbalance and before each sample preparation. Here we present the results for samples with a stoichiometry $\mathrm{Cu}_{96.7} \mathrm{~V}_{3.3}$. After evaporation, the surface of the $\mathrm{VCu}$ alloy was capped with a few monolayers of copper in order to prevent possible oxidation. Afterwards the samples were transferred quickly under UHV into the analysis chamber which remained in the low $10^{-11}$ mbar during measurements. The magnetic characterization of the $\mathrm{VCu}$ alloys was performed using XMCD in total electron-yield mode on beamline ID08 of the European Synchrotron Radiation Facility (ESRF) at Grenoble. The applied magnetic field of $7 \mathrm{~T}$ was parallel to the incident $\mathrm{x}$-ray beam and its direction was reversed between two successive photon-energy scans. The polarization rate was $99.9 \%$ and the absence of any contaminants (in particular of magnetic contaminants $\mathrm{Cr}, \mathrm{Fe}, \mathrm{Ni}$, and Co) was carefully checked using XAS.

Figure 1 displays the $\mathrm{V} L_{2,3}$ XAS measured for $\mathrm{VCu}$ alloys prepared at different temperatures. For comparison also the spectrum of an oxidized alloy is shown. The spectral shape of the XAS changes significantly with deposition temperature. The peak intensity for the alloys is at lower photon energy than the oxidized sample as is expected from the chemical shift. The samples prepared at 250 and at $300 \mathrm{~K}$ display a dominant $L_{3}$ feature centered at 518.3 and 516.5 $\mathrm{eV}$, respectively, and the sample prepared at $270 \mathrm{~K}$ has both features present in the XAS.

The absorption signal at these energies corresponds to electronic transitions from the $2 p$ core level to empty $3 d$ states. The differences in the XAS reveal therefore the existence of distinct electronic structures, i.e., different $\mathrm{V}$ species that can be either magnetic or nonmagnetic. The latter ones can be separated out, because only the magnetic species will give an XMCD signal. The influence of the deposition tem- 


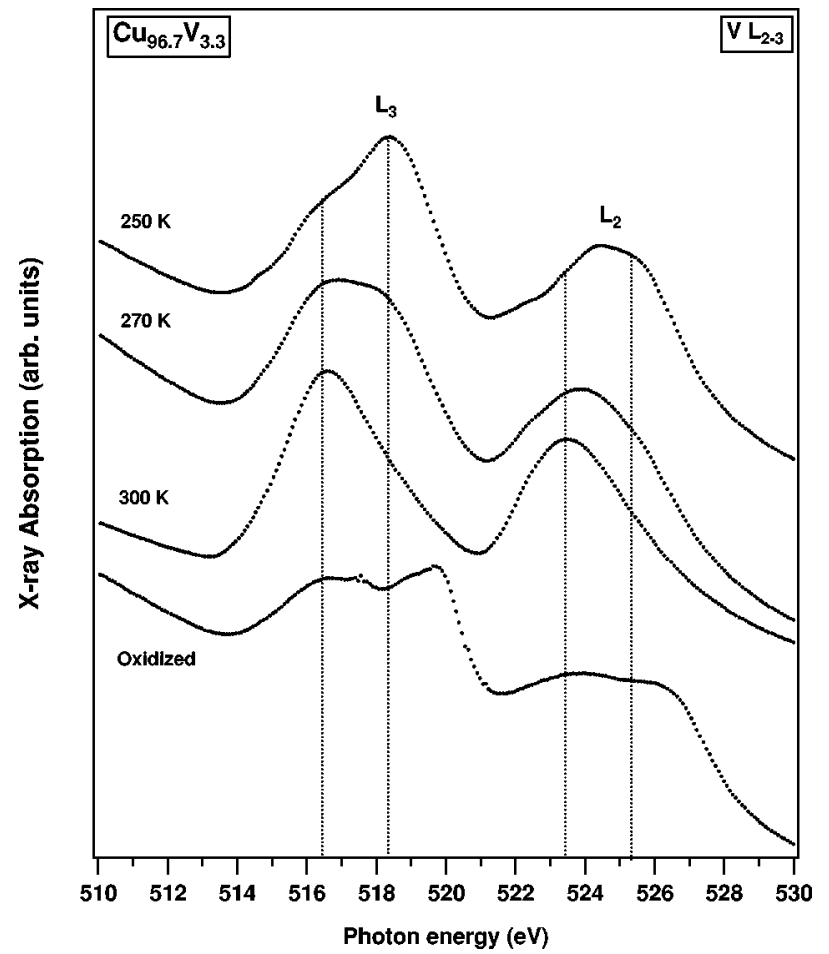

FIG. 1. Vanadium $L_{2,3}$ absorption spectra of $\mathrm{V}_{96.7} \mathrm{Cu}_{3.3}$ alloys prepared at different temperatures. The lower curve gives the spectrum for an oxidized alloy.

perature on the formation of different vanadium species can be understood in terms of vanadium migration. Even though for the dilute $\mathrm{V}$ concentration a random distribution would lead to single, isolated atoms, at increased temperatures the vanadium atoms are likely to migrate due to their thermal mobility and form clusters of larger sizes. The electronic structure and consequently the magnetic properties, which are very dependent on the size of the cluster, ${ }^{1,8,12-14}$ are expected to change strongly with the formation temperature of the alloy.

This behavior was further investigated by studying the magnetic properties of the samples prepared at different temperatures using XMCD. In Fig. 2 we present the absorption spectra (measured at $11 \mathrm{~K}$ ) corresponding to the sample prepared at $250 \mathrm{~K}$. The upper panel shows the absorption spectra measured with circularly polarized x-rays for opposite magnetic fields and the lower panel displays the resulting difference spectra, i.e., the XMCD $(\times 30)$ corresponding to the samples prepared at 250,270 , and $300 \mathrm{~K}$. The nonvanishing XMCD spectra corresponding to samples prepared at 250 and $270 \mathrm{~K}$ are a clear proof that the vanadium atoms have a magnetic moment in these samples. Interestingly, the sample prepared at $300 \mathrm{~K}$ as well as the oxidized sample did not give an observable magnetic signal. This is consistent with the aforementioned idea of migration of $\mathrm{V}$ atoms at high temperatures and the formation of bigger $\mathrm{V}$ clusters up to the limit of nonmagnetic bulk vanadium. It is interesting to note that the XMCD signal is relatively intense considering the small amount of V present in the samples. Furthermore, the spectral shape of the XMCD for the two magnetic samples was the same and resembled the shape measured for $\mathrm{V}$ de-

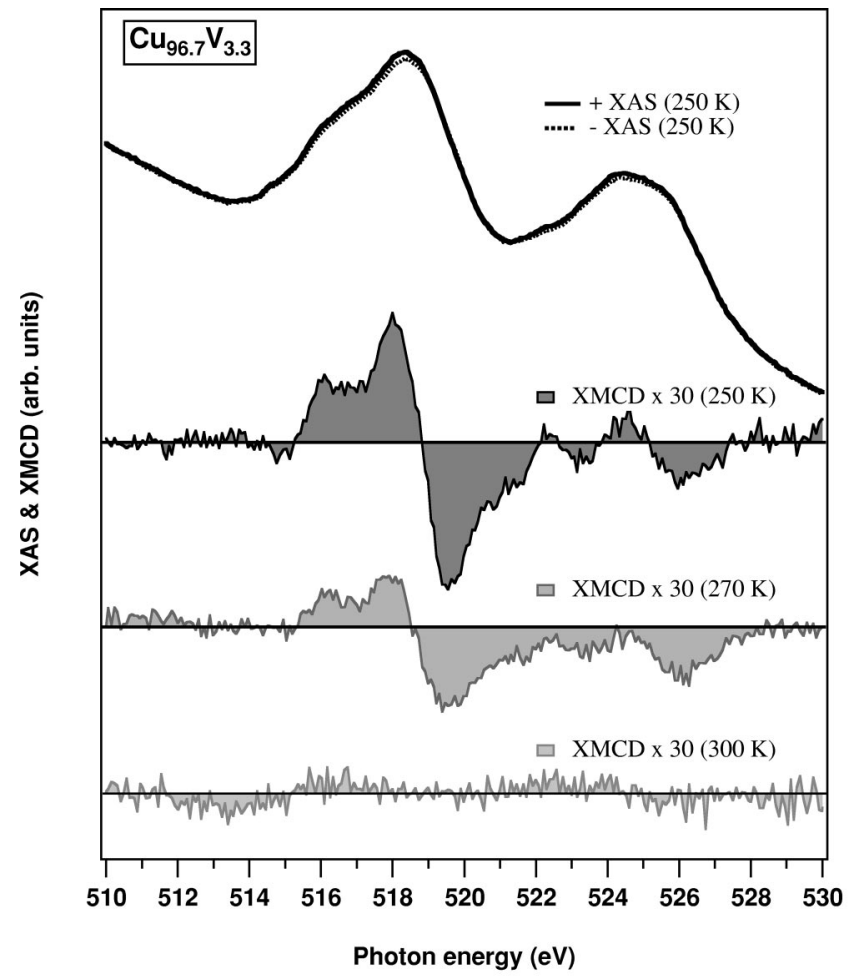

FIG. 2. V $L_{2,3}$ spectra measured at $11 \mathrm{~K}$ with circularly polarized $\mathrm{x}$ rays for opposite magnetic field directions and corresponding to the sample prepared at $250 \mathrm{~K}$. The resulting difference spectrum (XMCD) is presented together with those corresponding to the samples prepared at 270 and $300 \mathrm{~K}$ (measured at 11 and $12 \mathrm{~K}$, respectively).

posited on $\mathrm{Fe}(100),{ }^{11} \mathrm{VFe}$ alloy, ${ }^{22}$ and $\mathrm{Fe} / \mathrm{V}$ multilayers ${ }^{23}$ and superlattices. ${ }^{24,25}$ This is an important observation, since for those systems it is generally assumed that the magnetic moment on the vanadium atoms arises due to the induced polarization from neighboring Fe atoms. Our results rather suggest that-even though magnetic neighbors can participate in the polarization of vanadium atoms - this is not the only mechanism involved in the polarization of the vanadium atoms.

We now turn to Fig. 3 which shows the change in the V magnetic moment with temperature $T$ for the two magnetic alloys. Here we have extracted the magnetic moments from the XMCD signals assuming that the maximum of the normalized dichroic signal is proportional to the magnetic moment ${ }^{11}$ with the scaling factor obtained from a reference sample with known magnetic moment. ${ }^{24}$ This procedure is not perfect but probably the best one can do. In reality the XMCD signal integrated over the $L_{3}$ edge is proportional to $S_{z}+3 L_{z}+7 T_{z},{ }^{26}$ where $S_{z}, L_{z}$, and $T_{z}$ are the spin moment, orbital moment, and magnetic dipole moment, respectively. In atomiclike species, $L_{z}$ and $T_{z}$ can give a large contribution. ${ }^{27}$ In addition, for vanadium the second sum rule $^{28}$ cannot be applied due to the strong mixing between the $2 p_{3 / 2}$ and $2 p_{1 / 2}$ states which are separated by a relatively small $2 p$ spin-orbit splitting. The chosen procedure might include some systematic uncertainties, however, it gives vanadium magnetic moments that are in good agreement with 


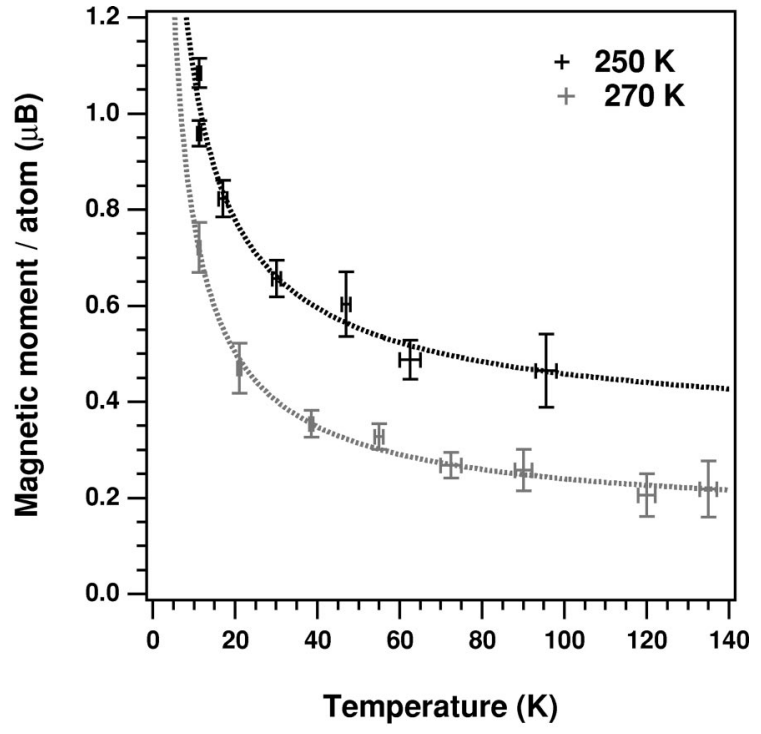

FIG. 3. Temperature dependence of the $\mathrm{V}$ magnetic moment $\mu$ obtained from the XMCD for the two magnetic alloys (those prepared at 250 and $270 \mathrm{~K}$ ). The dashed lines are guides to the eye.

previous results: the highest value for the atomic vanadium moment found in this work $\left(\mu \approx 1.1 \mu_{\mathrm{B}}\right)$ compares quite well with the magnetic moment measured in $\mathrm{V} / \mathrm{Fe}$ superlattices $^{24,25}\left(\sim 1\right.$ and $\sim 1.1 \mu_{\mathrm{B}}$, respectively), hyperfine $\mathrm{V}$ particles $^{8}\left(\sim 1.25 \mu_{\mathrm{B}}\right)$ in the dilute $\mathrm{V}$ limit, and the calculated value for the $\mathrm{VAu}_{4}$ alloy $^{17}\left(\sim 1.7 \mu_{\mathrm{B}}\right)$. It is clear that the formation temperature has a large influence on the $\mathrm{V}$ magnetic moment and that the sample prepared at lower temperature is more magnetic. Again this is can be understood in terms of the tendency of the vanadium atoms to form nonmagnetic clusters at higher temperatures. As a general trend, we observe a very strong vanadium susceptibility and a magnetic moment that decreases very rapidly with temperature in the low-temperature regime and then decays slowly for $T$ $>50 \mathrm{~K}$

The question that now arises is which mechanism is responsible for the strong variations observed in the atomic magnetic moment of vanadium. Due to the influence of the formation temperature on the magnetic moment, it seems reasonable to search for a thermodynamical explanation. This could be a mechanism where after deposition, and depending on the temperature, the vanadium atoms migrate and form bigger clusters with considerably reduced magnetic moments. Such a mechanism would explain the reduction of the magnetic moment with increasing temperature. The characteristics of such a change would be a strong modification of the XAS (such as for the different formation temperatures). However, after the $\mathrm{VCu}$ compound formation (i.e., after the deposition was performed) and during the measurements, when the temperature was tuned, no measurable change in the XAS could be observed indicating that the alloy is thermodynamically stable over the explored temperature range. Since the magnetic moment clearly increases with $1 / T$, it is tempting to assume a paramagnetic system where the alignment of the magnetic moments is determined by the magnetic field instead of by the thermal disorder. In order to

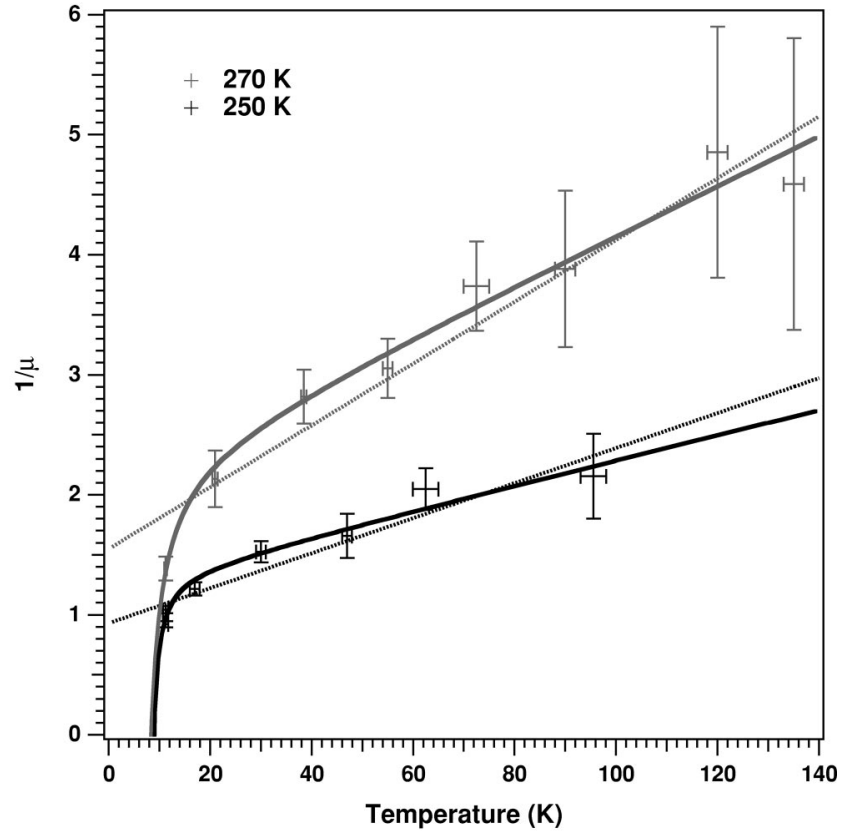

FIG. 4. Temperature dependence of $1 / \mu$ for the alloys prepared at 250 and $270 \mathrm{~K}$. Also displayed are the best fits assuming a CurieWeiss (dashed lines) and a ferrimagnetic (solid lines) law.

investigate the possible origin of the temperature dependence of the vanadium magnetic moment, we fitted the data points in Fig. 3 assuming a paramagnetic system. The results are displayed in Fig. 4 which shows the temperature dependence of $1 / \mu$ for the two magnetic samples. For a paramagnetic type of system, we can assume that $\mu$ is proportional to the magnetic susceptibility $\chi$. Therefore we have fitted the data points by a $1 / \chi$ law where $\chi$ is given by the Curie-Weiss law $\chi=C /(T-\theta)$. The corresponding fits are displayed by the dashed lines. It can be observed that these fits are rather poor, especially at low temperature. The values obtained for the Weiss temperature were close to $\theta \approx-60 \mathrm{~K}$. Apart from the fact that the best fits do not take correctly into account the data points at the lowest temperatures (note that these are the most accurate points), the resulting values for $\theta$ are too large to correspond to a paramagnetic or a superparamagnetic regime (usually $-10<\theta<10 \mathrm{~K}$ ). Since at high temperatures the alloy obeys a Curie-like law quite well, we tentatively fitted the data using a full Curie law in order to reduce the discrepancy at low temperatures between the data points and the fits (i.e., assuming that the approximation $k_{\mathrm{B}} T \gg g \mu_{\mathrm{B}} H$ is not valid $\left.{ }^{29}\right)$. However, the quality of the fits did not improve significantly, even not at low temperatures. The failure of the paramagnetic model at low temperatures is a clear indication that the magnetic moments are not isolated but that there must be magnetic interactions present in the alloy.

The magnetic interactions (which are more important at low temperatures) can be understood as an additional factor in the alignment of the magnetic moments (in absence of magnetic interactions the dominant factors are the magnetic field and the thermal disorder). From the data points given in Fig. 4, the antiferromagnetic and ferromagnetic systems can be excluded. In fact, for a ferromagnetic system $1 / \chi$ is nearly 
linear down to the Curie temperature and for an antiferromagnetic system, $1 / \chi$ should present a minimum at the Néel temperature and increase at lower temperatures. ${ }^{29-31}$ On the other hand, the fits given by solid lines were performed by assuming a ferrimagnetic law $1 / \chi=T / C+1 / \chi_{0}$ $-b /(T-\theta) .{ }^{30}$ As can be observed the resulting fits are much better than the previous ones, in particular at the lowest temperatures. Also the corresponding values, $\theta \approx 9 \mathrm{~K}$, are more realistic. This surprising result indicates that magnetic moments of different magnitudes are aligned antiparallel to each other. This could be due to the existence of clusters having different number of vanadium atoms, i.e., different magnetic moments and that arrange antiferromagnetically. Even though the local magnetic moments reflect the magnetic interactions between magnetic atoms, the XMCD technique is more an "atomic"-like probe of the magnetic properties than a "macroscopic" probe, and it is difficult to go beyond these observations and to conclude unambiguously the magnetic system of the $\mathrm{VCu}$ alloy. Nevertheless, these results clearly show that the $\mathrm{V}$ atoms feel a magnetic interaction; one that

*Electronic address: yves@imm.cnm.csic.es

†Electronic address: g.vanderlaan@dl.ac.uk

${ }^{1}$ B.V. Reddy, M.R. Pederson, and S.N. Khanna, Phys. Rev. B 55, R7414 (1997).

2 J.S. Moodera and R. Meservey, Phys. Rev. B 40, 8541 (1989).

${ }^{3}$ D.R. Grempel and S.C. Ying, Phys. Rev. Lett. 45, 1018 (1980).

${ }^{4}$ S. Bouarab et al., J. Magn. Magn. Mater. 104-107, 1765 (1992).

${ }^{5}$ C.L. Fu, A.J. Freeman, and S. Oguchi, Phys. Rev. Lett. 54, 2700 (1985)

${ }^{6}$ S. Ohnishi, C.L. Fu, and A.J. Freeman, J. Magn. Magn. Mater. 50, 161 (1985).

${ }^{7}$ C. Rau et al., Phys. Rev. Lett. 57, 2311 (1986).

${ }^{8}$ H. Akoh and A. Tasaki, J. Phys. Soc. Jpn. 42, 791 (1977).

${ }^{9}$ H. Beckmann, F. Ye, and G. Bergmann, Phys. Rev. Lett. 73, 1715 (1994)

${ }^{10}$ R.L. Fink et al., Phys. Rev. B 41, 10175 (1990).

${ }^{11}$ M. Finazzi et al., J. Magn. Magn. Mater. 165, 78 (1997).

${ }^{12}$ V.S. Stepanyuk et al., J. Magn. Magn. Mater. 165, 272 (1997).

${ }^{13}$ S.E. Weber et al., J. Phys.: Condens. Matter 9, 10739 (1997).

${ }^{14}$ K. Lee and J. Callaway, Phys. Rev. B 49, 13906 (1994). needs to be explored more deeply with complementary experimental techniques and theoretical calculations.

Summarizing, we have presented an XAS and XMCD study of $\mathrm{VCu}$ alloy. The deposition temperature of the alloy was found to be a determining factor for the formation of magnetic vanadium species which were evidenced by XAS and XMCD. The temperature dependence of the magnetic moment showed that the vanadium atoms do not follow a Curie-Weiss law but display rather a ferrimagnetic behavior. An extensive experimental and theoretical study will be required to fine-tune the set of parameters for the preparation of the vanadium copper alloys and to understand the magnetism of $\mathrm{VCu}$ alloys in order to optimize their properties for possible technological applications. We expect that the present study will stimulate new investigations on this type of magnetic material.

We acknowledge excellent technical assistance from the ESRF staff and in particular K. Larsson for his expertise and help during the experiment.

${ }^{15}$ S.L. Qiu, R.G. Jordan, A.M. Begley, X. Wang, Y Liu, and M.W. Ruckmen Phys. Rev. B 46, 13004 (1992).

${ }^{16}$ S. Blügel, Appl. Phys. A: Mater. Sci. Process. 63, 595 (1996).

${ }^{17}$ I. Galanakis et al., Phys. Rev. B 63, 172405 (2001).

${ }^{18}$ I.G. Batyrev et al., Phys. Rev. B 63, 172420 (2001).

${ }^{19}$ R. Robles et al., Phys. Rev. B 63, 172406 (2001).

${ }^{20}$ F. Song and G. Bergmann, Phys. Rev. Lett. 88, 167202 (2002).

${ }^{21}$ I.M.L. Billas, A. Châtelain, and W.A. De Heer, Science 265, 1682 (1994).

${ }^{22}$ M. Finazzi et al., J. Magn. Magn. Mater. 165, 373 (1997).

${ }^{23}$ G.R. Harp et al., Phys. Rev. B 51, 3293 (1995).

${ }^{24}$ M.M. Schwickert et al., Phys. Rev. B 57, 13681 (1998).

${ }^{25}$ A. Scherz et al., Phys. Rev. B 64, 180407 (2001).

${ }^{26}$ G. van der Laan, Phys. Rev. B 57, 112 (1998).

${ }^{27}$ P. Gambardella et al., Phys. Rev. Lett. 88, 047202 (2002).

${ }^{28}$ B.T. Thole et al., Phys. Rev. Lett. 68, 1943 (1992); P. Carra et al., ibid. 70, 694 (1993); C.T. Chen et al., ibid. 75, 152 (1995).

${ }^{29}$ N. W. Ashcroft and N. D. Mermin, Solid State Physics (Saunders College Publishing, New York, 1976).

${ }^{30}$ B. D. Cullity, Introduction to Magnetic Materials (AddisonWesley, New York, 1972).

${ }^{31}$ G. Burns, Solid State Physics (Academic Press, London, 1985). 